\section{An Intelligent Clinical Decision Support System for Predicting Acute Graft-versus- host Disease (aGvHD) following Allogeneic Hematopoietic Stem Cell Transplantation}

\author{
Cirruse Salehnasab ${ }^{1 \oplus}$, Abbas Hajifathali², Farkhondeh Asadi*, \\ Sayeh Parkhideh², Alireza Kazemi', Arash Roshanpoor³, Mah- \\ shid Mehdizadeh², Maria Tavakoli-Ardakani², Elham Roshandel ${ }^{4}$
}

\begin{abstract}
Background: Acute graft-versus-host disease (aGvHD) is a complex and often multisystem disease that causes morbidity and mortality in 35\% of patients receiving allogeneic hematopoietic stem cell transplantation (AHSCT).

Objective: This study aimed to implement a Clinical Decision Support System (CDSS) for predicting a GvHD following AHSCT on the transplantation day.

Material and Methods: In this developmental study, the data of 182 patients with 31 attributes, which referred to Taleghani Hospital Tehran, Iran during 2009-2017, were analyzed by machine learning (ML) algorithms which included XGBClassifier, HistGradientBoostingClassifier, AdaBoostClassifier, and RandomForestClassifier. The criteria measurement used to evaluate these algorithms included accuracy, sensitivity, and specificity. Using the machine learning developed model, a CDSS was implemented. The performance of the CDSS was evaluated by Cohen's Kappa coefficient.
\end{abstract}

Results: Of the 31 included variables, albumin, uric acid, C-reactive protein, donor age, platelet, lactate Dehydrogenase, and Hemoglobin were identified as the most important predictors. The two algorithms XGBClassifier and HistGradientBoostingClassifier with an average accuracy of $90.70 \%$, sensitivity of $92.5 \%$, and specificity of $89.13 \%$ were selected as the most appropriate ML models for predicting aGvHD. The agreement between CDSS prediction and patient outcome was $92 \%$.

Conclusion: ML methods can reliably predict the likelihood of aGvHD at the time of transplantation. These methods can help us to limit the number of risk factors to those that have significant effects on the outcome. However, their performance is heavily dependent on selecting the appropriate methods and algorithms. The next generations of CDSS may use more and more machine learning approaches.

Citation: Salehnasab C, Hajifathali A, Asadi F, Parkhideh S, Kazemi A, Roshanpoor A, Mehdizadeh M, Tavakoli-Ardakani M, Roshandel E. An Intelligent Clinical Decision Support System for Predicting Acute Graft-versus-host Disease (aGvHD) following Allogeneic Hematopoietic Stem Cell Transplantation. J Biomed Phys Eng. 2021;11(3):345-356. doi: 10.31661/jbpe.v0i0.2012-1244.

\section{Keywords}

Graft vs Host Disease; Decision Support Systems, Clinical; Machine learning; Classification

\section{Introduction}

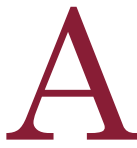
cute graft-versus-host disease (aGvHD) is a complex and often multisystem disease that causes morbidity and mortality in $35 \%-50 \%$ of patients receiving allogeneic hematopoietic stem cell transplantation (AHSCT) [1]. On the first 100 days after transplantation, donor $\mathrm{T}$ cells invade the host tissue and lead to dysfunction of
${ }^{1} \mathrm{PhD}$, Department of Health Information

Technology and Management, School of Allied

Medical Sciences, Sha-

hid Beheshti University

of Medical Sciences,

Tehran, Iran

${ }^{2} \mathrm{MD}$, Hematopoi-

etic Stem Cell Research

Center, Shahid Beheshti

University of Medical Sci-

ences, Tehran, Iran

${ }^{3} \mathrm{PhD}$, Department of

Computer Science,

Sama Technical and Vo-

cational Training College

Tehran Branch (Tehran),

Islamic Azad University

(IAU), Tehran, Iran

${ }^{4} \mathrm{PhD}$, Hematopoi-

etic Stem Cell Research

Center, Shahid Beheshti

University of Medical Sci-

ences, Tehran, Iran

*Corresponding author:

Farkhondeh Asadi

Department of Health

Information Technol-

ogy and Management,

School of Allied Medi-

cal Sciences, Shahid

Beheshti University of

Medical Sciences, Teh-

ran, Iran

E-mail: asadifar@sbmu. ac.ir

Received: 11 December 2020 Accepted: 10 April 2021 
the skin, gastrointestinal tract, and liver [1-4]. Given that it occurs at the stage of severe tissue damage, its diagnosis is late [5].

In recent years, biomarkers related to aGvHD have been considered as a tool in predicting the occurrence [5]. But the multiplicity of these biomarkers and the complexity of the various factors, contributing to the disease have made an accurate quick decision difficult. Besides, in previous studies [6-8]. The analyses performed on these biomarkers was univariate using classical statistical methods [9-11].

Since the 1960s, medical informatics experts have become interested in using clinical decision support systems (CDSS) to classify patient outcomes, reduce health-care costs, and alert physicians about the potential for dangerous medication interactions, resulting in the improvement of physicians' diagnostic process, and provide diagnostic suggestions, and also increase safety and quality of patient care [12-15].

CDSS is defined as "a computer system designed to impact clinician decision-making about individual patients at the point in time that these decisions are made" [13]. CDSSs are divided into two categories of knowledgebased and non-knowledge-based [13, 14]. In the knowledge-based type, the goal is to build a system that can simulate human thinking. These types of CDSSs use the knowledge as a rule or set of if-then rules in which they are specifically coded in clinical practice guidelines (CPG). Whereas, non-knowledge-based CDSSs use machine learning (ML) algorithms to extract knowledge [13].

Machine learning (ML) is a subset of artificial intelligence (AI) in which the algorithms, executing the prediction process extract the necessary knowledge from past experiences and/or find patterns in data [16-18]. ML is any process in which an algorithm is improved or "trained" by performing repetition on a training dataset to perform a task, usually a classification or identification $[16,19]$. The trained algorithms can then be evaluated by measuring its performance based on the test dataset $[17,19,20]$.

There are several learning methods in ML, one of the most widely used and the popular of which is supervised learning. The goal of a supervised learning algorithm is to use the dataset to produce a model, taking a feature vector $\mathrm{x}$ as an input and outputting information, resulting in deduction if the label for this feature vector [20].

The two major types of supervised learning are classification and regression. Examples of classification are ensemble methods, K-nearest neighbors, support vector machine, decision trees, random forest, neural networks and so on. Regression examples are linear regression and logistic regression [17, 19-21].

The ensemble is an ML concept in which the idea is to train multiple models using the same learning algorithm [22]. Ensemble algorithms are divided into two main types, including boosting and bagging. Ensemble methods include algorithms such as eXtreme Gradient Boosting classifier (XGBClassifier), AdaBoost classifier (AdaBoostClassifier), Histogram-based Gradient Boosting Classification Tree (HistGradientBoostingClassifier), and Random Forest classifier [20].

The XGBClassifier is a highly adaptable algorithm, working in most classifications. Boosting is a method, seeking to create a strong classifier based on weak classifiers. Weak and strong classification models mention to the correlation of outputs and actual class. By appending classifiers on top of each other iteratively, the next classifier can modify the errors of the previous one. This process is recurred until the training data set is accurately predicted [23].

The HistGradientBoostingClassifier has support for missing values. During training, the tree grower learns at each split point whether samples with missing values should go to the left or right child, based on the potential gain [19]. 
An Intelligent CDSS for Predicting aGvHD

An AdaBoost classifier (AdaBoostClassifier) is one of the most popular algorithms for building robust classifiers with linear combinations of member classifiers. The member classifiers are chosen to minimize the errors in each iteration during the training process [24].

RandomForestClassifier synthesizes several randomized decision trees and gathers their predictions by averaging. In settings where the number of variables is much greater than the number of observations, this method has shown excellent performance [25].

Pre-occurrence prediction by these algorithms helps physicians to identify high-risk patients and reduce health care costs by performing time-consuming treatment interventions [26].

Previous studies have shown that neural network algorithms, support vector machine, naive bayes, K-nearest neighbors, regression, decision trees, and ensemble methods have been used to predict aGvHD [27]. Although in recent years the decision trees and ensemble methods have been given more attention for predicting aGvHD, there is no evidence that these algorithms are successfully used in the clinical setting [28-30]. Therefore, this study aimed to design, implement, and validate a clinical decision support system using ensemble methods to predict aGvHD following AHSCT on transplant day.

\section{Material and Methods}

Data Source, Study Roadmap, and Tools

In a developmental study, 31 variables [27] (which were classified into two groups: baseline and biomarker), which could potentially affect the transplantation outcome, were gathered on the day of transplantation from $190 \mathrm{pa}-$ tients who received AHSCT in Taleghani Hospital, Tehran, Iran, from 2009 to 2017. Then the CDSS was designed and implemented using Python programming language in four stages as pre-processing, learning, evaluation, and CDSS implementation as is shown in the below roadmap diagram (Figure 1).

\section{Pre-processing}

Imputing missing value

In this phase, the raw data were imputed using the following two processes:

1 - Records and variables with missing values (greater than 50\%) were excluded from the dataset.

2- The missing values of continuous and discrete variables were replaced separately with mean and mode in each class, respectively.

Under-sampling

Under-sampling methods normalize the distribution of all classes by decreasing the number of majority class records in the imbalanced

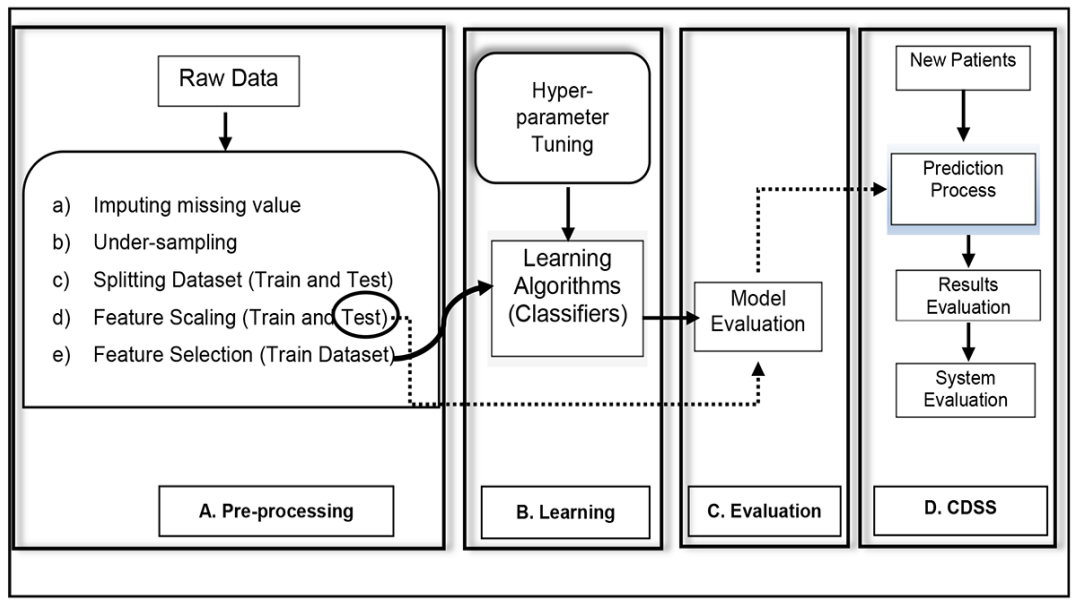

Figure 1: Roadmap for building clinical decision support systems based on machine learning. 
dataset [31]. An imbalanced class distribution will have one or more classes with few samples (the minority classes) and one or more classes with many samples (the majority classes). In this study, the RandomUnderSampler method was used to decrease the number of majority class records [32]. RandomUnderSampler is a fast and simple method to balance the patient's dataset by randomly choosing a subset of data for the targeted classes.

Data Splitting

In this phase, patients' datasets were divided into training and testing sets with a ratio of $70 \%$ and $30 \%$, respectively.

\section{Feature Scaling}

In this phase, the training and test data sets were scaled separately using the normalizing method (Equation 1) [32]. The numerical values of the data set are between zero and one.

1) $\mathrm{X}_{\text {normalized }}=\left(\mathrm{X}-\mathrm{X}_{\min }\right) /\left(\mathrm{X}_{\max }-\mathrm{X}_{\min }\right)$

\section{Feature Selection}

In this phase, the Boruta algorithm, which is a type of wrapper method feature selection, was used to select the most important predictors of aGvHD prediction. This method, using RandomForestClassifier algorithm, identifies important features of the dataset as unbiased and stable [33].

\section{Learning}

Hyperparameters are parameters, governing the learning process, but they are not the part of the learning process. Besides, they have a great impact on the performance and results of modeling ML algorithms [34]. Adjustment of these hyperparameters is considered as an optimization problem and their search is usually done manually using methods such as randomized parameter optimization with $\mathrm{k}$ fold cross-validation method (RandomizedSearchCV) [19, 35]. In the present study, the RandomizedSearchCV method was used to optimize the hyperparameters of four ML algorithms, including XGBClassifier, HistGradientBoostingClassifier, AdaBoostClassifier, and RandomForestClassifier.

\section{Evaluation}

After modeling the ML algorithms, their performance was evaluated using the accuracy, sensitivity, specificity, F-measure, and AUC (area under the curve) criteria (Equations 2 to 5) [36].

$$
\begin{aligned}
& \text { 2) } \\
& \text { 3) } \quad \text { Sensitivity }(T P R)=\frac{T P+T N}{T P+F N} \\
& \text { 4) } \\
& \text { 5pecificity }(T N R)=\frac{T N}{T N+F P} \\
& \text { 5) } \quad F \text {-measure }=\frac{2 \times T P}{2 \times T P+F P+F N}
\end{aligned}
$$

A ROC chart is defined by false positive rate (FPR) and true positive rate (TPR) as $\mathrm{x}$ and $\mathrm{y}$ axes, respectively, depicting relative trade-offs between true positive (TP) and false-positive (FP) [36].

Where TP is the number of actual patients, predicted correctly to have aGvHD. TN is the number of non-patients, predicted correctly not to have aGvHD. False-positive (FP) is the number of non-patients, predicted incorrectly to have aGvHD, and false-negative $(\mathrm{FN})$ is the number of patients, predicted incorrectly not to have aGvHD [36].

\section{CDSS}

After selecting the most appropriate ML models, a CDSS was designed and implemented using Python programming language and MySQL database management system (Figure 1 Part D).

Then performance of CDSS was evaluated by calculating the agreement between CDSS prediction and the actual patient outcome after 100 days of transplantation, using Cohen's Kappa coefficient and transplant data 30 patients, receiving AHSCT in 2018 [37].

\section{Results}

\section{a. Patient Characteristics}

Table 1 presents the most significant variables for predicting aGvHD. 
Table 1: The dataset variables and their descriptions.

\begin{tabular}{|c|c|c|c|c|}
\hline Type & Row & Variable & Description & Role \\
\hline \multirow{12}{*}{ 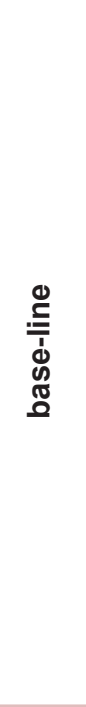 } & 1 & Patient Gender & & Input \\
\hline & 2 & Donor Gender & & Input \\
\hline & 3 & Donor-Patient Gender & & Input \\
\hline & 4 & Patient Blood group & & Input \\
\hline & 5 & Donor Blood group & & Input \\
\hline & 6 & Delivery & The process of giving birth for Donor. & Input \\
\hline & 7 & Marital Status & & Input \\
\hline & 8 & Smoking & & Input \\
\hline & 9 & Blood group Compatibility & $\begin{array}{l}\text { Donor and recipient have the same blood group antigens and plasma } \\
\text { antibodies. }\end{array}$ & Input \\
\hline & 10 & Donor recipient relationship & $\begin{array}{l}\text { The relation between donor and patient gender including Related and } \\
\text { Sibling. }\end{array}$ & Input \\
\hline & 11 & Patient Age & & Input \\
\hline & 12 & Donor Age & & Input \\
\hline \multirow{19}{*}{ 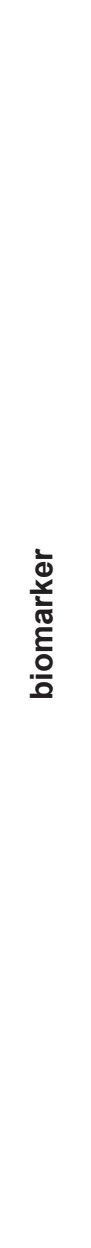 } & 1 & Prophylaxis Regimen & Regimen use for the prevention of a specific disease. & Input \\
\hline & 2 & Chemotherapy Regimen & $\begin{array}{l}\text { Regimen 1-3: Myeloablative is an intensive conditioning regimen to destroy } \\
\text { the bone marrow cells. Regimen 4: Reduced intensity conditioning that } \\
\text { uses less chemotherapy and radiation than the Regimen 1-3. }\end{array}$ & Input \\
\hline & 3 & Diagnosis & & Input \\
\hline & 4 & Complete Remission & $\begin{array}{l}\text { Including: tests, physical exams, and scans show that all signs of cancer } \\
\text { are gone. }\end{array}$ & Input \\
\hline & 5 & $\begin{array}{l}\text { Radiothrapypre Bone Marrow } \\
\text { Transplantation }\end{array}$ & The treatment of disease with ionizing radiation. & Input \\
\hline & 6 & White Blood Cells & & Input \\
\hline & 7 & Platelet count & & Input \\
\hline & 8 & lactate dehydrogenase (LDH) & & Input \\
\hline & 9 & $\begin{array}{l}\text { cluster of differentiation } 3 \\
\text { (CD3) }\end{array}$ & & Input \\
\hline & 10 & $\begin{array}{l}\text { cluster of differentiation } 34 \\
\qquad(\mathrm{CD} 34)\end{array}$ & The CD34 antigen identifies on a myeloid leukemia cell line. & Input \\
\hline & 11 & mononuclear cell (MNC) & & Input \\
\hline & 12 & Diagnosis to Transplantation & $\begin{array}{l}\text { The time between disease diagnosis and hematopoietic stem cell trans- } \\
\text { plantation }\end{array}$ & Input \\
\hline & 13 & Patient Body mass index & & Input \\
\hline & 14 & Donor Body mass index & & Input \\
\hline & 15 & Hemoglobin & & Input \\
\hline & 16 & Creatinine & & Input \\
\hline & 17 & Uric Acid & & Input \\
\hline & 18 & Albumin & & Input \\
\hline & 19 & C-Reactive Protein (CRP) & & Input \\
\hline & & $\begin{array}{l}\text { Acute graft-versus-host } \\
\text { disease (aGvHD) }\end{array}$ & & Target \\
\hline
\end{tabular}


b. Pre-processing

After discarding incomplete patient records, the patient dataset was reduced to 182 patients (71 case-patients diagnosed with aGvHD vs. 111 control patients who did not experience aGvHD). As a result of under-sampling, the final number of scaled (normalized) patient records was 142 patients ( 71 cases vs. 71 controls), of which $70 \%$ (99 patients) were selected for the training dataset and 30\% (43 cases) for the testing dataset.

The results of feature selection showed that of the 31 included variables, albumin, uric acid, C-Reactive Protein, donor age, platelet, Lactate Dehydrogenase, and Hemoglobin were identified as the seven most important predictors of aGvHD (Table 2) of which, albumin had the highest importance.

\section{c. Predictive performance}

The results of tuning the hyperparameters of ML algorithms are presented in Table 3.

The evaluation results of ML models based on the test data set are shown in Figure 2 and Table 4. Based on the evaluation criteria including, accuracy, sensitivity, specificity, Fmeasure, and area under the curve (AUC), the two algorithms XGBClassifier (eXtreme Gradient Boosting Classifier) and HistGradientBoostingClassifier had the best performance. According to the mean of evaluation criteria, the XGBClassifier algorithm with 90.82 and the lowest number of false negative and false

Table 2: The most important predictors of acute graft-versus-host disease

\begin{tabular}{cc} 
Feature & Importance \\
\hline Albumin & 0.409 \\
\hline Uric Acid & 0.151 \\
\hline C-Reactive Protein & 0.148 \\
\hline Donor age & 0.085 \\
\hline Platelet & 0.081 \\
\hline Lactate Dehydrogenase & 0.071 \\
\hline Hemoglobin & 0.055
\end{tabular}

Table 3: Results of optimized hyperparameters of machine learning algorithms

\begin{tabular}{cc} 
Classifier & $\begin{array}{c}\text { Best F-measure } \\
\%\end{array}$ \\
\hline 'XGBClassifier & 94 \\
\hline $\begin{array}{c}\text { HistGradientBoosting- } \\
\text { Classifier }\end{array}$ & 90 \\
\hline AdaBoostClassifier & 90 \\
\hline RandomForestClassifier & 95
\end{tabular}

*eXtreme Gradient Boosting classifier

positive had the best performance (Table 4).

\section{d. CDSS}

Using the machine learning developed the model, a CDSS was designed and implemented, which is accessible via the https://agprcdss.ir/ (Figure 3). The agreement between

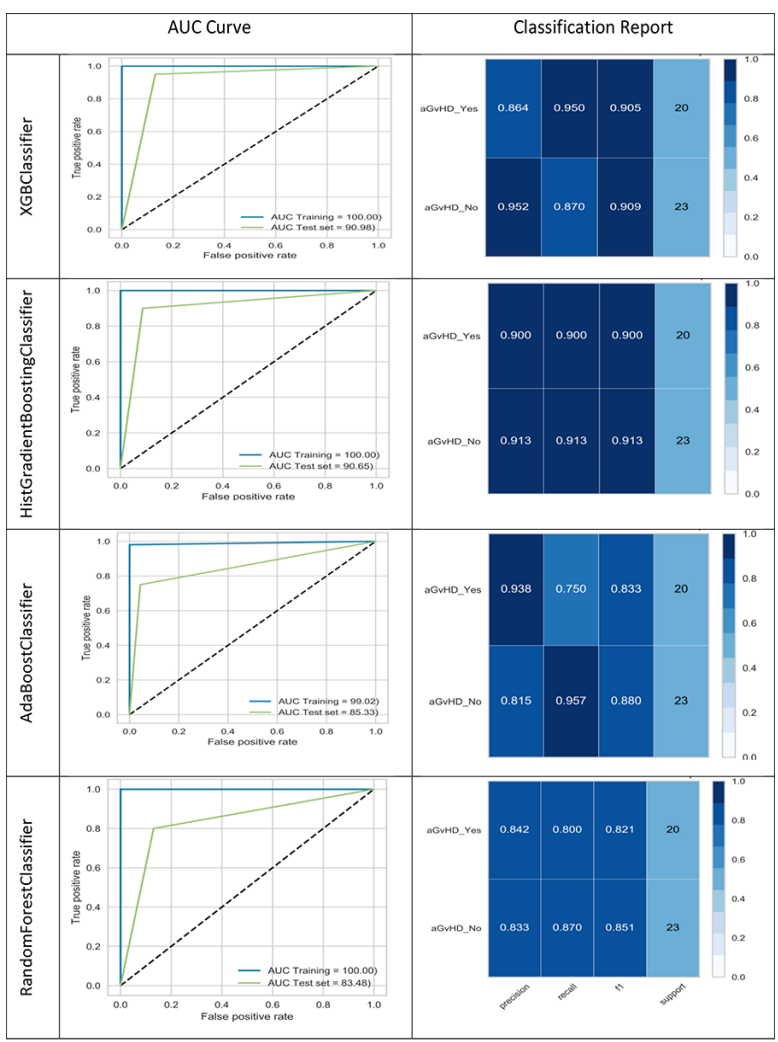

Figure 2: Results of classification report and Area under the curve (AUC) curve of machine learning models. 
CDSS prediction and the actual outcome that occurred within 100 days after AHSCT was $92 \%$.

\section{Discussion}

In this study, we designed and implemented the AGPRC (Acute GvHD Prediction Transplant Day CDSS) for predicting the likelihood of aGvHD on transplantation day. Considering the most important aGvHD predictors and ML classification models, in the following lines, we have discussed some important aspects of this study.

I. The most important predictors for aGvHD

Biomarkers play a key role in predicting
aGvHD as they help oncologists to identify patients who are at higher risk for aGvHD, and to select appropriate pre and post transplantation care plans for them. In this study, seven variables were identified as the most important factors associated with aGvHD on the transplantation day. These variables included albumin, uric acid, CRP, donor age, platelet, LDH, and hemoglobin.

In our study, the relative importance of albumin in predicting aGvHD was about $41 \%$. Similarly, previous studies have also emphasized on the importance of the albumin level for predicting aGvHD [38-40], and low amounts of albumin alone and without dependence on other predictors affect overall mortality of aGvHD patients [41].

Table 4: Results of performance evaluation of machine learning models

\begin{tabular}{cccccccc} 
Row & Classifier & Accuracy & Sensitivity & Specificity & F-measure & AUC & Mean \\
\hline $\mathbf{1}$ & XGBClassifier & 90.70 & 95.00 & 86.96 & 90.48 & 90.98 & 90.82 \\
\hline $\mathbf{2}$ & $\begin{array}{c}\text { HistGradientBoost- } \\
\text { ingClassifier }\end{array}$ & 90.70 & 90.00 & 91.30 & 90.00 & 90.65 & 90.53 \\
\hline $\mathbf{-}$ & Average 1 and 2 & 90.70 & 92.50 & 89.13 & 90.24 & 90.82 & - \\
\hline $\mathbf{3}$ & AdaBoostClassifier & 86.05 & 75.00 & 95.65 & 83.33 & 85.33 & 85.07 \\
\hline $\mathbf{4}$ & $\begin{array}{c}\text { RandomForest- } \\
\text { Classifier }\end{array}$ & 83.72 & 80.00 & 86.96 & 82.05 & 83.48 & 83.24
\end{tabular}

AUC: Area under the curve, XGBClassifier: eXtreme Gradient Boosting classifier

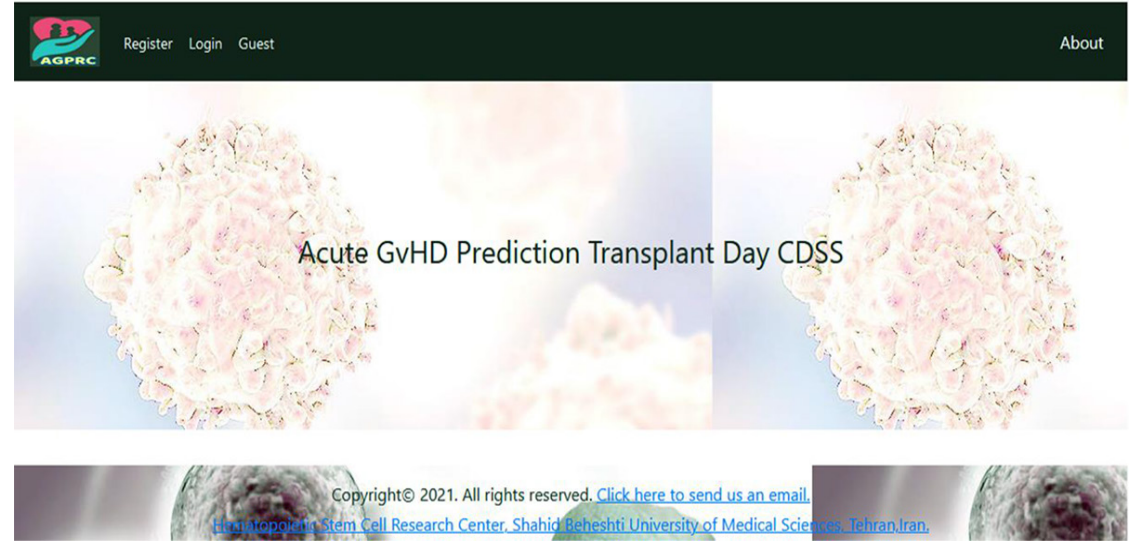

Figure 3: Graphical user interface of the clinical decision support system. 
The second predictor for aGvHD is uric acid with the importance of $15.1 \%$. In previous studies, this variable has been cited as a strong immunological risk signal. Low levels of this predictor, especially on the day of transplantation, increase the likelihood of aGvHD [42, 43].

The third predictor for predicting aGvHD is CRP with the importance of $14.8 \%$. High levels of this predictor in patients increase the risk of aGvHD, especially grade II to IV, asymptomatic death, and decreased overall survival [44-48].

The fourth predictor for aGvHD is the donor age with the importance of $8.5 \%$. In studies related to AHSCT, donor age is considered as an important predictor [49], and one of the appropriate predictors that can be achieved easily and without cost.

The fifth predictor for aGvHD is platelets with the importance of $8.1 \%$. Previous studies have emphasized that to prevent the likelihood of aGvHD in AHSCT patients, it is essential to maintain platelet counts above $10,000 \mathrm{~mm}^{3}$ [50].

The sixth predictor for aGvHD is LDH with the importance of $7.1 \%$. Studies have shown that low levels of LDH and high levels of serum cyclosporine reduce the likelihood of aGvHD [51]. In some previous studies, this variable has been presented as one of the important predictors $[10,52]$.

The seventh predictor for predicting aGvHD is hemoglobin with the importance of 5.5\%. Previous studies have emphasized that to prevent the likelihood of aGvHD in AHSCT patients, maintaining a hemoglobin level above 8 to $9 \mathrm{~g} / \mathrm{dL}$ is essential. Therefore, red blood cells and platelets are injected continuously in these patients [50].

According to the literature review, each one of previous studies has focused on the importance of a marker based on the diagnosis of aGvHD. Thus, the impact of the combination of these significant factors on aGvHD detection is reported for the first time in this study.
II. Selected machine learning models

After identifying the most important predictors, the algorithm modeling process was performed using the optimization of their hyperparameters. Based on the evaluation criteria of accuracy, sensitivity, specificity, AUC, F-measure, and the average of these criteria, XGBClassifier model and HistGradientBoostingClassifier had the best performance.

In previous studies, ML algorithms have been mainly used in laboratory settings, not in clinical practice. In the present study, the selected and tuned ML models were used as the inference engine of a CDSS to predict aGvHD in the transplantation unit of the target hospital.

In 2015, a study by Cocho et al, [53] aimed at using different ML algorithms to diagnose aGvHD by gene expression data, used support vector machine (SVM), shrinkage discriminant analysis (SDA), K-nearest neighbors (KNN) algorithms without tuning their hyperparameters. The reported sensitivity, specificity and AUC were $100 \%, 92.9 \%$ and $99.5 \%$ for SVM, 92.9\%, 92.9\% and 95.9\% for SDA and $92.9 \%, 92.9 \%$ and $92.9 \%$ for $\mathrm{KNN}$, respectively. The ML models presented in this study had very good performance evaluation criteria, but there are three main criticisms for this study as follows: 1) this study aimed to diagnose aGvHD only based on gene expression data. 2) These ML models have not been tested in a clinical setting, and 3) the system cannot predict aGvHD before the patient goes through transplantation because the study was designed in such way, measured after transplantation.

In 2018, Arai et al, [28] conducted a study entitled "Predicting aGvHD following $\mathrm{AH}$ SCT using an ML algorithm" using the ADTree without the hyper-parameter optimization method. The reported AUC for grades 2-4 aGvHD was $61.6 \%$ and for grades 3-4 was $62.3 \%$. This study aimed to develop ML models to accurately predict grades 2 to 4 of 
An Intelligent CDSS for Predicting aGvHD

aGvHD, However, the performance of their models was poor. In contract, in our study, all performance measures of the selected ML models had values over $90 \%$, which demonstrates a much better overall performance than Arai et al, study.

In 2018, Lee et al, [29] conducted a study entitled as "Predicting the absolute risk of aGvHD following AHSCT" using the ensemble method without optimizing the hyperparameters of the employed algorithms. The reported AUC was in the range of $61.3 \%$ to $64 \%$ for these ensemble models. Despite the fact that in the present study, the models are also of the ensemble type, because of the hyperparameter tuning, the performance was much better compared to the study conducted by Lee et al.

In 2020, Tang et al, [54] conducted a study entitled "Predicting aGvHD using Machine Learning and Longitudinal Vital Signs Data from Electronic Health Records" using logistic regression without hyper-parameters optimization methods. The reported AUC for grades 2-4 aGvHD was $65.9 \%$. This study, like a few other studies $[28,29,53]$, was performed to diagnose aGvHD after transplantation. Compared to the ML models of the present study, the model proposed in the study of Tang et al, has a lower performance, and has not been used in a clinical setting.

Comparing the performance evaluation criteria of XGBClassifier and HistGradientBoostingClassifier with ML model presented in previous studies [28, 29, 53, 54], it seems that the use of these ML models in CDSS to predict aGvHD in the process of modifying the care plan of patients who received AHSCT can be useful and effective. Thus, we designed and developed a CDSS and applied it in the transplantation unit of the target hospital to predict aGvHD on the day of transplantation.

\section{CDSS performance evaluation}

In terms of developing aGvHD, there was $92 \%$ agreement between the CDSS predic- tion outcome and the actual patient outcome that was measured 100 days after the AHSCT transplantation.

Given that the criteria of the average evaluation of the ML models used in this CDSS were $91 \%$, it seems that this CDSS had acceptable performance.

\section{Conclusion}

According to the current results and previous research, it is obvious that training a model based on the aggregation of the most significant features achieves the better performance in comparison with generating a model concerning each important feature, separately.

In this study, seven variables were identified as the most important factors associated with aGvHD on the transplantation day. These variables included albumin, uric acid, CRP, donor age, platelet, LDH, and hemoglobin. Ensembled Machine learning methods can reliably predict the likelihood of aGvHD at the time of transplantation. These methods can help us to limit the number of risk factors to those that have the significant effects on the outcome. However, their performance is heavily dependent on selecting the appropriate methods and algorithms. Future studies should focus on determining the most appropriate aGvHD predictive models.

\section{Acknowledgment}

The authors would like to thank the reviewers for their valuable comments suggestions to improve the quality of the paper. This study was part of a $\mathrm{PhD}$ project conducted at Shahid Beheshti University of Medical Sciences, Tehran, Iran (Grant Number: IR.SBMU. REC.1397.128).

\section{Conflict of Interest}

None

\section{References}

1. Gopalakrishnan R, Jagasia M. Pathophysiology and Management of Graft-Versus-Host Disease. In Hematopoietic Cell Transplantation for Malignant 
Conditions. Elsevier Health Sciences; 2019. p. 30119. doi: 10.1016/B978-0-323-56802-9.00022-5.

2. Ferrara JL, Levine JE, Reddy P, Holler E. Graft-versus-host disease. Lancet. 2009;373(9674):155061. doi: 10.1016/S0140-6736(09)60237-3. PubMed PMID: 19282026. PubMed PMCID: PMC2735047.

3. Blazar BR, Murphy WJ, Abedi M. Advances in graft-versus-host disease biology and therapy. Nat Rev Immunol. 2012;12(6):443. doi: 10.1038/ nri3212. PubMed PMID: 22576252. PubMed PMCID: PMC3552454.

4. Ball L, Egeler RM. Acute GvHD: pathogenesis and classification. Bone Marrow Transplant. 2008;41(S2):S58. doi: 10.1038/bmt.2008.56. PubMed PMID: 18545246.

5. Ali AM, DiPersio JF, Schroeder MA. The Role of Biomarkers in the Diagnosis and Risk Stratification of Acute Graft-versus-Host Disease: A Systematic Review. Biology of blood and marrow transplantation. Journal of the American Society for Blood and Marrow Transplantation. 2016;22(9):1552-64. doi: 10.1016/j.bbmt.2016.04.022. PubMed PMID: 27158050. PubMed PMCID: PMC5599102.

6. Betts B, Anasetti C, Pidala J. Biomarkers for GVHD prognosis. Lancet Haematol. 2015;2(1):e4-5. doi: 10.1016/S2352-3026(14)00040-4. PubMed PMID: 26687427.

7. Berger M, Signorino E, Muraro M, Quarello P, Biasin $E$, Nesi $F$, et al. Monitoring of TNFR1, IL-2R $\alpha, H G F$, CCL8, IL-8 and IL-12p70 following HSCT and their role as GVHD biomarkers in paediatric patients. Bone Marrow Transplant. 2013;48(9):1230. doi: 10.1038/bmt.2013.41. PubMed PMID: 23584443.

8. Gimondi S, Dugo M, Vendramin A, Bermema A, et al. Circulating miRNA panel for prediction of acute graft-versus-host disease in lymphoma patients undergoing matched unrelated hematopoietic stem cell transplantation. Exp Hematol. 2016;44(7):62434. doi: 10.1016/j.exphem.2016.03.005. PubMed PMID: 27013207.

9. Levine JE, Braun TM, Harris AC, Holler E, et al. Blood and Marrow Transplant Clinical Trials Network. A prognostic score for acute graft-versushost disease based on biomarkers: a multicentre study. Lancet Haematol. 2015;2(1):e21-9. doi: 10.1016/S2352-3026(14)00035-0. PubMed PMID: 26687425. PubMed PMCID: PMC4340092.

10. Paczesny S, Krijanovski OI, Braun TM, Choi SW, et al. A biomarker panel for acute graft-versushost disease. Blood. 2009;113(2):273-8. doi: 10.1182/blood-2008-07-167098. PubMed PMID: 18832652. PubMed PMCID: PMC2615645.
11. Levine JE, Logan BR, Wu J, Alousi AM, et al. Acute graft-versus-host disease biomarkers measured during therapy can predict treatment outcomes: a Blood and Marrow Transplant Clinical Trials Network study. Blood. 2012;119(16):3854-60. doi: 10.1182/blood-2012-01-403063. PubMed PMID: 22383800. PubMed PMCID: PMC3335389.

12. Shortliffe EH. Biomedical Informatics: the Science and the pragmatics. In Biomedical informatics. London: Springer; 2014. p. 3-37. doi: 10.1007/978-1-4471-4474-8_1.

13. Berner ES. Clinical decision support systems. New York: Springer; 2007.

14. Alther M, Reddy CK. Clinical Decision Support Systems. Healthcare Data Analytics; 2015.

15. El-Sappagh SH, El-Masri S. A distributed clinical decision support system architecture. Journal of King Saud University - Computer and Information Sciences. 2014;26(1):69-78. doi: 10.1016/j. jksuci.2013.03.005.

16. Raschka S, Mirjalili V. Python machine learning. Birmingham, UK: Packt Publishing Ltd; 2017.

17. Géron A. Hands-on machine learning with ScikitLearn and TensorFlow: concepts, tools, and techniques to build intelligent systems 1st Edition. O’Reilly Media, Inc; 2017.

18. Asadi F, Salehnasab C, Ajori L. Supervised Algorithms of Machine Learning for the Prediction of Cervical Cancer. J Biomed Phys Eng. 2020;10(4):513. doi: 10.31661/jbpe.v0i0.19121027. PubMed PMID: 32802799. PubMed PMCID: PMC7416093.

19. Géron A. Hands-On Machine Learning with ScikitLearn, Keras, and TensorFlow: Concepts, Tools, and Techniques to Build Intelligent Systems 2st Edition. O'Reilly Media, Inc; 2019.

20. Alpaydin E. Introduction to machine learning. MIT press; 2014.

21. Rezaianzadeh A, Dastoorpoor M, Sanaei M, et al. Predictors of length of stay in the coronary care unit in patient with acute coronary syndrome based on data mining methods. Clinical Epidemiology and Global Health. 2020;8(2):383-8. doi: 10.1016/j.cegh.2019.09.007.

22. Kumar A, Jain M. Ensemble Learning for Al Developers. Berkeley, CA: Apress; 2020. doi: 10.1007/978-1-4842-5940-5.

23. Sang V, Yano S, Kondo TJS. On-Body Sensor Positions Hierarchical Classification. Sensors. 2018;18(11):3612. doi: 10.3390/s18113612.

24. An T-K, Kim M-H, editors. A new diverse AdaBoost classifier. International Conference on Artificial In- 
An Intelligent CDSS for Predicting aGvHD

telligence and Computational Intelligence; Sanya, China: IEEE; 2010. doi: 10.1109/AICI.2010.82.

25. Biau G, Scornet EJT. A random forest guided tour. Test. 2016;25(2):197-227. doi: 10.1007/s11749016-0481-7.

26. Obermeyer Z, Emanuel EJ. Predicting the futurebig data, machine learning, and clinical medicine. N Engl J Med. 2016;375(13):1216. doi: 10.1056/ NEJMp1606181. PubMed PMID: 27682033. PubMed PMCID: PMC5070532.

27. Salehnasab C, Hajifathali A, Asadi F, et al. Machine Learning Classification Algorithms to Predict aGvHD following Allo-HSCT: A Systematic Review. Methods of Information in Medicine. 2019;58(06):20512. doi: 10.1055/s-0040-1709150. PubMed PMID: 32349154.

28. Arai Y, Kondo T, Fuse K, Shibasaki Y, Masuko M, et al. Using a machine learning algorithm to predict acute graft-versus-host disease following allogeneic transplantation. Blood Adv. 2019;3(22):362634. doi: 10.1182/bloodadvances.2019000934. PubMed PMID: 31751471 . PubMed PMCID: PMC6880900.

29. Lee C, Haneuse S, Wang HL, Rose S, et al. Prediction of absolute risk of acute graft-versus-host disease following hematopoietic cell transplantation. PLoS One. 2018;13(1):e0190610. doi: 10.1371/ journal.pone.0190610. PubMed PMID: 29346409. PubMed PMCID: PMC5773230.

30. Paun 0, Phillips T, Fu P, Novoa RA, et al. Cutaneous complications in hematopoietic cell transplant recipients: impact of biopsy on patient management. Biol Blood Marrow Transplant. 2013;19(8):1204-9. doi: 10.1016/j.bbmt.2013.05.006. PubMed PMID: 23688396.

31. Barkai K. Accomplishment Classifier with Machine Learning. San Francisco, CA 94103: Minerva Schools at KGl; 2017.

32. Swamynathan M. Mastering machine learning with python in six steps: A practical implementation guide to predictive data analytics using python. Berkeley, CA: Apress; 2019. doi: 10.1007/978-14842-4947-5.

33. Kursa MB, Rudnicki WR. Feature selection with the Boruta package. J Stat Softw. 2010;36(11):1-3. doi: 10.18637/jss.v036.i11.

34. Claesen M, De Moor B. Hyperparameter search in machine learning. ArXiv:1502.02127v2. 2015.

35. Mantovani RG, Rossi AL, Vanschoren J, Bischl $B$, De Carvalho AC, editors. Effectiveness of random search in SVM hyper-parameter tuning. International Joint Conference on Neural Networks (IJCNN); Killarney, Ireland: IEEE: 2015. doi
10.1109/IJCNN.2015.7280664.

36. Sokolova M, Japkowicz N, Szpakowicz S, editors. Beyond accuracy, F-score and ROC: a family of discriminant measures for performance evaluation. Australasian joint conference on artificial intelligence; Berlin, Heidelberg: Springer; 2006. doi: 10.1007/11941439_114.

37. Artstein R, Poesio M. Inter-coder agreement for computational linguistics. Computational Linguistics. 2008;34(4):555-96. doi: 10.1162/coli.07034-R2.

38. Ayuk F, Bussmann L, Zabelina T, Veit R, et al. Serum albumin level predicts survival of patients with gastrointestinal acute graft-versus-host disease after allogeneic stem cell transplantation. Ann Hematol. 2014;93(5):855-61. doi: 10.1007/s00277-0131957-0. PubMed PMID: 24248672.

39. Harada K, Sekiya N, Konishi T, Nagata A, et al. Predictive implications of albumin and C-reactive protein for progression to pneumonia and poor prognosis in Stenotrophomonas maltophilia bacteremia following allogeneic hematopoietic stem cell transplantation. BMC Infect Dis. 2017;17(1):638. doi: 10.1186/s12879-017-2745-6. PubMed PMID: 28938875. PubMed PMCID: PMC5610439.

40. Gadallah HA, Khalaf MH, Mohamed HS. Pretransplant C-reactive protein, ferritin, albumin, and platelet count as prognostic biomarkers of hematopoietic stem cell transplantation outcome in hematological malignancies. The Egyptian Journal of Haematology. 2018;43(2):76. doi: 10.4103/ejh. ejh_47_17.

41. Artz AS, Logan BR, Zhu X, et al. Pre-Transplant C-Reactive Protein (CRP), Ferritin and Albumin As Biomarkers to Predict Transplant Related Mortality (TRM) after Allogeneic Hematopoietic Cell Transplant (HCT). Blood. 2014;124 (21):422. doi: 10.1182/blood.V124.21.422.422.

42. Haen SP, Eyb V, Mirza N, Naumann A, Peter A, et al. Uric acid as a novel biomarker for bone-marrow function and incipient hematopoietic reconstitution after aplasia in patients with hematologic malignancies. J Cancer Res Clin Oncol. 2017;143(5):75971. doi: 10.1007/s00432-017-2348-z. PubMed PMID: 28210842.

43. Ostendorf BN, Blau O, Uharek L, Blau IW, Penack 0 . Association between low uric acid levels and acute graft-versus-host disease. Ann Hematol. 2015;94(1):139-44. doi: 10.1007/s00277-0142180-3. PubMed PMID: 25172456.

44. Artz AS, Wickrema A, Dinner S, Godley LA, et al. Pretreatment $\mathrm{C}$-reactive protein is a predictor for outcomes after reduced-intensity allogeneic hema- 
topoietic cell transplantation. Biol Blood Marrow Transplant. 2008;14(11):1209-16. doi: 10.1016/j. bbmt.2008.08.004. PubMed PMID: 18940674. PubMed PMCID: PMC2668514.

45. Fuji S, Kim SW, Fukuda T, Mori S, Yamasaki S, et al. Preengraftment serum C-reactive protein (CRP) value may predict acute graft-versus-host disease and nonrelapse mortality after allogeneic hematopoietic stem cell transplantation. Biol Blood Marrow Transplant. 2008;14(5):510-7. doi: 10.1016/j. bbmt.2008.02.008. PubMed PMID: 18410893.

46. Jordan KK, Christensen IJ, Heilmann C, Sengeløv $H$, Müller KG. Pretransplant C-reactive protein as A prognostic marker in allogeneic stem cell transplantation. Scand J Immunol. 2014;79(3):206-13. doi: 10.1111/sji.12137. PMID: 24313319.

47. Minculescu L, Kornblit BT, Friis LS, Schiødt I, et al. C-Reactive Protein Levels at Diagnosis of Acute Graft-versus-Host Disease Predict SteroidRefractory Disease, Treatment-Related Mortality, and Overall Survival after Allogeneic Hematopoietic Stem Cell Transplantation. Biol Blood Marrow Transplant. 2018;24(3):600-7. doi: 10.1016/j. bbmt.2017.10.025. PubMed PMID: 29074374.

48. Sato $M$, Nakasone $H$, Oshima $K$, Ishihara $Y$, et al. Prediction of transplant-related complications by C-reactive protein levels before hematopoietic SCT. Bone Marrow Transplant. 2013;48(5):698702. doi: 10.1038/bmt.2012.193. PubMed PMID: 23042494.

49. Baygan A, Aronsson-Kurttila W, Moretti G, et al. Safety and Side Effects of Using Placenta-Derived Decidual Stromal Cells for Graft-versus-Host Dis- ease and Hemorrhagic Cystitis. Front Immunol. 2017;8:795. doi: 10.3389/fimmu.2017.00795. PubMed PMID: 28744284. PubMed PMCID: PMC5504152.

50. Govindan R. The Washington manual of oncology. Lippincott Williams \& Wilkins; 2008.

51. Song MK, Chung JS, Seol YM, et al. Influence of lactate dehydrogenase and cyclosporine a level on the incidence of acute graft-versus-host disease after allogeneic stem cell transplantation. J Korean Med Sci. 2009;24(4):555-60. doi: 10.3346/ jkms.2009.24.4.555. PubMed PMID: 19654932. PubMed PMCID: PMC2719215.

52. Amini M, Kazemnejad A, Rasekhi A, Zayeri F, et al. Application of latent class analysis in diagnosis of graft-versus-host disease by serum markers after allogeneic haematopoietic stem cell transplantation. Sci Rep. 2020;10(1):3633. doi: 10.1038/ s41598-020-60524-2. PubMed PMID: 32108153. PubMed PMCID: PMC7046680.

53. Cocho L, Fernández I, Calonge M, Martínez V, et al. Gene Expression-Based Predictive Models of Graft Versus Host Disease-Associated Dry Eye. Invest Ophthalmol Vis Sci. 2015;56(8):457081. doi: 10.1167/iovs.15-16736. PubMed PMID: 26200497.

54. Tang S, Chappell GT, Mazzoli A, Tewari M, et al. Predicting Acute Graft-Versus-Host Disease Using Machine Learning and Longitudinal Vital Sign Data From Electronic Health Records. JCO Clin Cancer Inform. 2020;4:128-135. doi: 10.1200/ CCI.19.00105. PubMed PMID: 32083957. PubMed PMCID: PMC7049247. 